\title{
Evaluación de un protocolo de diagnóstico rápido de síndrome coronario agudo
}

Evaluation of a rapid protocol to diagnose acute coronary syndrome

Than M y col Lancet 2011;377:1077-1084.

\section{Objetivos}

Validar la seguridad de un protocolo de diagnóstico rápido (PDR) de síndrome coronario agudo (SCA) en pacientes con dolor torácico (DT) sugestivo de SCA para poder darlos de alta luego de dos horas de observación en la central de emergencias (CE).

\section{Diseño, lugar y pacientes}

Cohorte prospectiva desarrollada en 14 CE de Asia-Pacífico. Incluyó mayores de 18 años que consultaron por DT compatible con SCA de más de cinco minutos de duración en quienes se había decidido la medición de biomarcadores. Criterios de exclusión: SCA con supradesnivel del segmento ST, causa de DT diferente de SCA y derivación a otro centro.

Descripción de las pruebas evaluadas y medición de los resultados principales

EI PDR consistió en la combinación de un cuestionario preestablecido basado el puntaje de riesgo TIMI, la presencia de cambios electrocardiográficos (ECG) y la medición rápida de troponina, mioglobina y CK-mb al ingreso y a las dos horas. El paciente fue considerado de bajo riesgo cuando todos los parámetros fueron negativos. Los médicos tratantes desconocían los resultados del cuestionario y de las enzimas del PDR.

Fueron resultados principales los eventos adversos cardíacos (EAC) mayores a los 30 días: muerte, paro cardíaco, revascularización de emergencia, shock cardiogénico, arritmia ventricular que requiriera intervención médica, bloqueo aurículo ventricular de alto grado e IAM.

\section{Resultados principales}

De los 3.853 pacientes elegibles fueron enrolados 3.651 y 3.582 completaron el seguimiento, registrándose un EAC en $421(11,8 \%)$ y siendo el IAM sin supradesnivel del ST el más frecuente (86,2\% de los casos). El PDR clasificó a 352 (9,8\%) individuos como de bajo riesgo de EAC, de los cuales tres $(0,9 \%)$ sufrieron un evento durante el seguimiento. Ver tabla 1.

Tabla 1: medidas de desempeño de las distintas herramientas diagnósticas evaluadas en el protocolo.

\begin{tabular}{|c|c|c|c|c|}
\hline & $\begin{array}{l}\text { Sensibilidad* } \\
\text { (\% e IC95\%) }\end{array}$ & $\begin{array}{l}\text { Especificidad* } \\
\text { (\% e IC95\%) }\end{array}$ & $\begin{array}{l}\text { Coef. de probabilidad } \\
\text { positivo }(\mathrm{LR}+)^{*}\end{array}$ & $\begin{array}{l}\text { Coef. de probabilidad } \\
\text { negativo (LR-)* }\end{array}$ \\
\hline Electrocardiograma (ECG) & $35,2(30,7$ a 9,8$)$ & $72,2(70,6$ a 3,7$)$ & $1,3(1,1$ a 1,5$)$ & $0,9(0,8$ a 1,0$)$ \\
\hline Enzimas & $82,9(79,0$ a 6,2$)$ & $56,0(54,3$ a 57,7$)$ & $1,9(1,8$ a 2,0$)$ & $0,3(0,3$ a 0,4$)$ \\
\hline TIMI & $96,7(94,5$ a 8,0$)$ & $17,6$ (16,3 a 18,9$)$ & $1,2(1,1$ a 1,2$)$ & $0,2(0,1$ a 0,3$)$ \\
\hline Enzimas + ECG & $88,8(85,5$ a 1,5$)$ & $43,0(41,2$ a 44,7$)$ & $1,6(1,5$ a 1,6$)$ & $0,3(0,2$ a 0,3$)$ \\
\hline TIMI + ECG & $98,1(96,3$ a 9,0$)$ & $14,6(13,4$ a 15,8$)$ & $1,1(1,1$ a 1,2$)$ & $0,1(0,1$ a 0,3$)$ \\
\hline PDR & $99,3(97,9$ a 9,8$)$ & $11,0(10,0$ a 12,2$)$ & $1,1(1,1$ a 1,3$)$ & $0,1(0,0$ a 0,2$)$ \\
\hline
\end{tabular}

\section{Conclusiones}

Este PDR identifica pacientes de bajo riesgo de EAC a 30 días, que podrían ser dados de alta precozmente de la CE, para disminuir el tiempo de observación y disminuir las internaciones por DT.

Palabras claves: dolor de pecho, infarto agudo de miocardio, troponina, unidad de dolor, enzimas cardíacas. Key words: Chest pain, acute myocardial infarction, troponin, chest pain unit, cardiac enzymes. Fuente de financiamiento: Alere Medical, Queensland Emergency Medicine Research Foundation and National Health, Medical Research Council, Christchurch Cardio-Endocrine Research Group, Medquest Jaya Global, Science International, Bio Laboratories Pte, National Heart Foundation of New Zealand, y Progressive Group.

\section{Comentario}

Las unidades de dolor torácico (UDT) surgen para identificar a los individuos en riesgo de sufrir un SCA y evitar internaciones innecesarias ${ }^{1}$. Sin embargo, aun no contamos con un protocolo con sensibilidad y especificidad suficientes para alcanzar dichos objetivos. Si bien este trabajo reportó una sensibilidad para la detección de SCA de 99,3\%, había excluido a quienes habían consultado por síntomas atípicos de SCA, lo que no es menor ya que representan una de las principales causas de error diagnóstico ${ }^{2}$. Por otro lado, su baja especificidad puede determinar un mayor número de internaciones, como reportaron Goddacre $S$ y col. ${ }^{3}$.

A pesar de las publicaciones de los últimos años ${ }^{4,5}$ esta estrategia de manejo ultra rápido no se ha difundido en la práctica clíni-

\section{Ignacio Bluro [ Servicio de Cardiología del Hospital Italiano de Buenos Aires ignacio.bluro@ hospialitaliano.org.ar ]}

Recibido el 22/06/2011 y aceptado el 01/09/2011 ca, probablemente debido a que el DT puede ser un síntoma de patologías diferentes al SCA (síndrome aórtico agudo, tromboembolismo de pulmón) que pueden cursar sin cambios ECG ni enzimáticos y haciendo que muchos de estos pacientes sean sometidos a métodos diagnósticos adicionales antes de su alta de la $\mathrm{CE}^{6}$.

\section{Conclusiones del comentador}

Los SCA pueden cursar con muy diferentes presentaciones, por lo que se deben contemplar otros diagnósticos diferenciales que también ponen en riesgo la vida, lo que hace difícil imaginar cómo llevar a la práctica la implementación de un PDR como el descripto.

Bluro I. Evaluación de un protocolo de diagnóstico rápido de síndrome coronario agudo. Evid Act Pract Ambul. Jul-Set 2011;14(3):99. Comentado de: Than $\mathrm{M}$ y col. A 2-h diagnostic protocol to assess patients with chest pain symptoms in the Asia-Pacific region (ASPECT); a prospective observational validation study. Lancet 2011;377:1077-1084. PMID: 21435709.

\section{Referencias}

1. Farkouh M y col. A clinical trial of a chest-pain observation unit for patients with unstable angina. Chest Pain Evaluation in the Emergency Room (CHEER) Investigators. N Engl J Med. 1998 Dec 24;339(26):1882-8.

2. Pope J, y col. Missed diagnoses of acute cardiac ischemia in the emergency department. N Engl J Med. 2000;342:1163-1170.
3. Goddacre S y col. The Randomised Assessment of Treatment using Panel Assay of Cardiac Markers (RATPAC) trial: a randomised controlled trial of point-of-care cardiac markers in the emergency 3. Goddacre Sy col. The Randomised $A$

4. Ng S y col. Ninety-Minute Accelerated Critical Pathway for Chest Pain Evaluation. Am J Cardiol 2001;88:611-617.

5. McCord P. y col. Point-of-Care Testing of Myoglobin and Troponin I Ninety-Minute Exclusion of Acute Myocardial Infarction By Use of Quantitative. Circulation 2001;104:1483-1488.

6. Rogers I y col. Usefulness of comprehensive cardiothoracic computed tomography in the evaluation of acute undifferentiated chest discomfort in the emergency department (CAPTURE). Am J Cardiol. 2011 Mar 1;107:643-50. 